\title{
Verhalten von Windkraftanlagen mit doppelt speisenden Asynchrongeneratoren (DFIG) bei Kurzschlüssen und anderen Netzfehlern
}

\author{
R. Schürhuber, B. R. Oswald, L. Fickert, J. Fortmann
}

\begin{abstract}
Motiviert durch die Energiewende steuert die elektrische Energieversorgung in vielen Ländern auf eine Erzeugungsstruktur zu, welche von leistungselektronisch gekoppelten Erzeugungseinheiten, allen voran Windkraftwerken (WKW) und Photovoltaikanlagen, dominiert wird. Dies hat zur Folge, dass sich die Auslegung der elektrischen Betriebsmittel sowie die Planung und der Betrieb der Netze zunehmend an den Eigenschaften solcher Anlagen ausrichtet. Wegen ihrer technischen Komplexität stellen dabei Windkraftwerke (WKW) mit doppelt speisenden Generatoren (double-feed induction generator bzw. doubly-fed induction generator, DFIG) eine besondere Herausforderung dar, da bei ihnen sowohl die durch die Physik bestimmten Eigenschaften der rotierenden elektrischen Maschine als auch das maßgeblich durch Steuerung und Regelung beeinflusste Verhalten des Umrichters zum Tragen kommt. In diesem Artikel wird das Verhalten der aktuellen Technologie von DFIG bei Kurzschlüssen und anderen Netzfehlern beschrieben, der Stand der Technik in der für Anlagenauslegung wichtigen Kurzschlussnorm erläutert sowie ein neuer Vorschlag für die Nachbildung der WKW mit DFIG in dieser Norm unterbreitet. Weiters wird auf spezielle Anforderungen an den Netzschutz eingegangen.
\end{abstract}

Schlüsselwörter: Kurzschlussberechnung; IEC 60909-0; DIN EN 60909-0; doppelt gespeister Generator; doppelt gespeiste Asynchronmaschine; Windkraftanlage; DFG; DFIG; unterspannungsgesteuerte Überstromauslösung; 51V; unterspannungsgesteuerter Anrege-Selbsthaltung; Reserveschutz; weak infeed

\section{Behavior of wind turbines with doubly-fed induction generators (DFIG) in the event of short circuits and other grid faults.}

Motivated by the energy transition, the electrical energy supply is heading for a generating structure in many countries dominated by generating units connected to the grid with power electronics and with wind turbines and photovoltaic systems leading the way. As a result, the design of the electrical equipment as well as the planning and operation of the power system are increasingly based on the properties of such units. Because of their technical complexity, wind turbines with doubly-fed (induction) generators (DFIG) in particular represent a challenge. This is due to both the properties of the rotating electrical machine coming into play. These are determined by physics and the behavior of the converter that is significantly influenced by control. In this article, the behavior of the current DFIG technology in case of short-circuit faults and other network faults is described. Additionally, a state-of-the-art treatment of DFIGs in the short-circuit standard - which is essential for system design - is explained and a new proposal for treatment in this standard is made. Furthermore, special requirements for network protection are formulated and discussed.

Keywords: short-circuit calculation; IEC 60909-0; DIN EN 60909-0; doubly-fed generator; doubly-fed induction generator; DFG; DFIG; doubly-fed induction machine; wind turbine; undervoltage-controlled overcurrent release; $51 \mathrm{~V}$; overcurrent protection with undervoltage release; undervoltage-controlled seal-in circuit with overcurrent starter

Eingegangen am 31. Juli 2020, angenommen am 16. September 2020, online publiziert am 12. Oktober 2020 (C) The Author(s) 2020

\section{Einleitung}

Die Erzeugung elektrischer Energie aus Windkraftwerken hat im letzten Jahrzehnt weltweit immens an Bedeutung zugenommen und in vielen Ländern einen Durchdringungsgrad erreicht, in dem sie einen signifikanten Einfluss auf die Energieversorgung ausübt, sowohl im Hinblick auf die installierte Leistung als auch auf den Anteil an erzeugter Jahresenergie. So ist die installierte Leistung seit 2010 um rund 100..200\% angewachsen (Abb. 1).

In Zahlen zeigt sich die Bedeutung der Windleistung für die elektrische Energieerzeugung aus den Werten in Tab. 1 (Stand 2017/18):

Aufgrund der Relevanz solcher Anlagen für die elektrische Energieversorgung ist es unumgänglich, Windkraftanlagen bei Planung und Betrieb der Netze gewissenhaft zu integrieren und das Verhal- ten von Netzen mit dominant umrichterbasierter Einspeisung, wie sie zukünftig großflächig zu erwarten ist, sicher zu beherrschen. Ein wichtiger Aspekt dabei ist das Verhalten bei Netzfehlern, denn ein sicheres und vorhersehbares Beherrschen solcher Situationen ist entscheidend für einen stabilen, sicheren und zuverlässigen Netzbetrieb. Beim Fehlerverhalten elektrischer Erzeugungsanlagen besteht ein prinzipieller Unterschied, ob die Einspeisung unmittelbar über ei-

Schürhuber, Robert, Technische Universität Graz, Graz, Austria

(E-Mail: robert.schuerhuber@tugraz.at); Oswald, Bernd R., DKE/K121, Frankfurt am Main, Deutschland; Fickert, Lothar, Technische Universität Graz, Graz, Austria; Fortmann, Jens, Hochschule für Technik und Wirtschaft, Berlin, Deutschland 


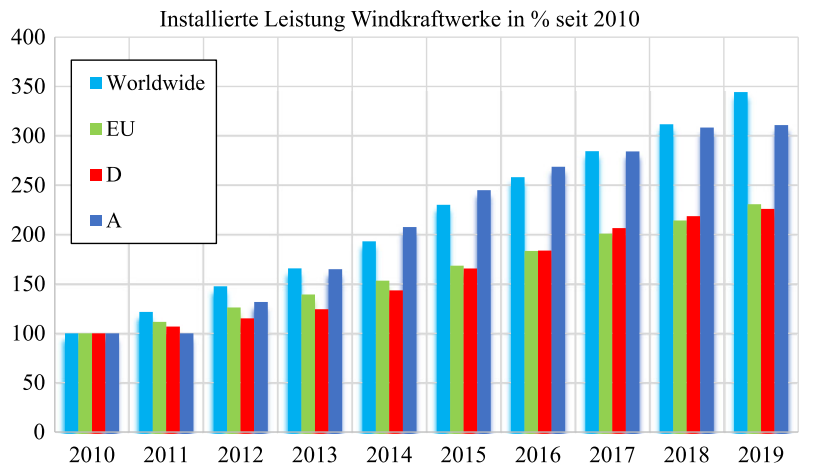

Abb. 1. Entwicklung der installierten Windleistung seit 2010 [1, 2], Balken von links nach rechts: Worldwide, EU, D, A

Tab. 1. Installierte Windleistung und Anteil der durch Windkraftanlagen erzeugten Energie an der gesamten elektrischen Energieerzeugung $[1,2]$

\begin{tabular}{llll}
\hline & $\begin{array}{l}\text { Installierte } \\
\text { Windleistung }\end{array}$ & $\begin{array}{l}\text { Prozent der } \\
\text { installierten } \\
\text { Gesamtleistung }\end{array}$ & $\begin{array}{l}\text { Prozent der } \\
\text { elektrischen } \\
\text { Jahresarbeit }\end{array}$ \\
\hline Weltweit & $564 \mathrm{GW}$ & $7 \%$ & $5 \%$ \\
EU & $183 \mathrm{GW}$ & $18 \%$ & $14 \%$ \\
D & $59 \mathrm{GW}$ & $27 \%$ & $26 \%$ \\
A & $3,1 \mathrm{GW}$ & $12 \%$ & $8,9 \%$ \\
\hline
\end{tabular}

ne rotierende elektrische Maschine und einen Transformator erfolgt, oder ob noch eine leistungselektronische Komponente, ein sog. Umrichter, zwischen Erzeugungseinheit und Netzanschluss geschaltet ist. Während bei der ersten Variante das Fehlerverhalten direkt durch die Physik und damit die Konstruktion der Maschine bestimmt ist, übt im zweiten Fall die Steuerung und Regelung des Umrichters einen entscheidenden Einfluss aus. Eine besondere Herausforderung für die Modellierung und auch die Auslegung des Schutzes stellen in dieser Hinsicht doppeltspeisende Asynchrongeneratoren (DFIG) dar, da bei ihnen die Eigenschaften beider Anschlussvarianten im Fehlerfall zum Tragen kommen: Unmittelbar nach Fehlereintritt dominiert die Physik der rotierenden elektrischen Maschine das Fehlerverhalten, kurze Zeit später passt dann der Umrichter die Ausgangswerte des Stroms entsprechend der implementierten Regelung an. In diesem Artikel wird das prinzipielle Verhalten von DFIG im Hinblick auf seine Einbindung ins Netz analysiert und es werden daraus Schlussfolgerungen gezogen, wie solche Anlagen aus aktueller Sicht unter verschiedenen praktischen Aspekten zu behandeln sind. Konkret wird erörtert:

- Verhalten der DFIG im Normalbetrieb und im Fehlerfall.

- Behandlung der DFIG in den Kurzschlussnormen IEC 600909-0 und DIN EN 60909-0 (VDE 0102).

- Netzanschlussbedingungen sowie Aspekte des Netzschutzes.

DFIG finden breite Anwendung in Windkraftwerken (WKW), dabei wird zur effizienten Nutzung des vorhandenen Winds die Drehzahl den aktuellen Windverhältnissen angepasst. Zur Anpassung der variablen Generatordrehzahl (und damit der variablen Frequenz) an die (weitgehend) feste Netzfrequenz kommen aktuell hauptsächlich zwei verschiedene Technologien zum Einsatz: Bei WKW mit Vollumrichtern (FSC, full size converter, Type 4 gemäß [3]) ist ein Umrichter mit zumindest der vollen Maschinenwirkleistung zwischen Generator und Transformator geschaltet. Aus Netzsicht ist dabei die
Tab. 2. Vor- und Nachteile der Windkraftwerke mit Umrichtern sowie der DFIG

\begin{tabular}{|c|c|}
\hline WKW mit DFIG (Type 3) & WKW mit FSC (Type 4) \\
\hline $\begin{array}{l}\text { Vorteile: } \\
\text { - Umrichterleistung geringer, } \\
\text { dadurch Kostenvorteil }\end{array}$ & $\begin{array}{l}\text { Vorteile: } \\
\text { - Getriebelose Konstruktion } \\
\text { möglich, dadurch erhöhte } \\
\text { Zuverlässigkeit } \\
\text { - Einfachere Regelung, vor al- } \\
\text { lem im Fehlerfall }\end{array}$ \\
\hline $\begin{array}{l}\text { Nachteile: } \\
\text { - Eingeschränkter Drehzahlbe- } \\
\text { reich des Generators ( } \approx 30 \% \\
\text { Schlupf) } \\
\text { - Aufwändigere Regelung nö- } \\
\text { tig um den Fehlerfall zu be- } \\
\text { herrschen }\end{array}$ & $\begin{array}{l}\text { Nachteile: } \\
\text { - Getriebelose Anlage im Ver- } \\
\text { gleich größer und schwerer, } \\
\text { damit teurer }\end{array}$ \\
\hline $\begin{array}{l}\text { Hauptanwendungen: } \\
\text { - hauptsächlich Onshore } \\
\text { WKW, Leistung bis } \approx 6 \mathrm{MW} \\
\text { - Drehzahlvariable Pumpspei- } \\
\text { cherkraftwerke großer } \\
\text { Leistung }(\approx>150 \mathrm{MW}) \text {. }\end{array}$ & $\begin{array}{l}\text { Hauptanwendungen: } \\
\text { - Onshore und Offshore WKW, } \\
\text { Leistung bis } \approx 10 \mathrm{MW} \\
\text { - Drehzahlvariable Pumpspei- } \\
\text { cherkraftwerke mittlerer } \\
\text { Leistung }(\approx 80 . .150 \mathrm{MW})\end{array}$ \\
\hline
\end{tabular}

Maschine vom Netz weitgehend entkoppelt und der Umrichter bestimmt praktisch ausschließlich das Fehlerverhalten. Die zweite weitverbreitete Variante ist die DFIG (Type 3 gemäß [3]), hier ist der Umrichter in den Rotorkreis des Generators geschaltet, siehe Abb. 5. Stationär bestimmt der Umrichter den Betrieb der Anlage, im transienten Bereich muss jedoch auch das Verhalten des Generators berücksichtigt werden. In Tab. 2 sind Vor- und Nachteile der beiden Varianten zusammengefasst.

In Abb. 2 sind die Kurzschlussstromverläufe der wichtigsten Erzeugungsanlagen prinzipiell dargestellt. Der Kurzschlussstrom der Synchronmaschine weist ein auf null abklingendes Gleichstromglied sowie ein auf einen stationären Wert abklingendes Wechselstromglied auf. Charakteristisch ist, dass der Kurzschlussstrom eine hohe Anfangsspitze besitzt ( $\approx 6 . .8$-facher Bemessungsstrom). Die Spannungsregelung des Generators hat auf den Anfangsverlauf des Kurzschlussstromes kaum Einfluss, sie beginnt erst nach einigen Perioden wirksam einzugreifen. Ähnliche Stromverläufe, jedoch mit geringeren Zeitkonstanten, finden sich auch bei direkt, d. h. nicht über Umrichter, angeschlossene Asynchrongeneratoren.

Beim FSC hingegen ist das Kurzschlussverhalten gänzlich verschieden. Schon nach sehr kurzer Zeit (1..2 Perioden) hat die UmrichterRegelung den Kurzschlussstrom vollständig unter Kontrolle und liefert gemäß den Netzanforderungen Blindstrom. Aufgrund der fehlenden Überlastfähigkeit der im Umrichter als Schalter eingesetzten Halbleiterventile muss der Kurzschlussstrom limitiert werden und ist geringer als bei der Synchronmaschine, er liegt in der Größenordnung des Bemessungsstroms des Umrichters, typ. bei etwa 1,2..1,4 $\cdot$ Iru.

Bei der DFIG ist ein anderes Verhalten zu erkennen: Anfangs dominieren von der Maschine verursachte Stromspitzen, die rasch abklingen und dann von einem durch den von der Umrichter-Regelung eingestellten Kurzschlussstrom abgelöst werden.

\section{Nachbildung der Windkraftwerke mit DFIG in den Normen zur Berechnung der Kurzschlussströme}

Die Berechnung der Kurzschlussströme nach den Normen IEC 60909-0 und DIN VDE 0102 [4, 5] stellt ein vereinfachtes Berech- 

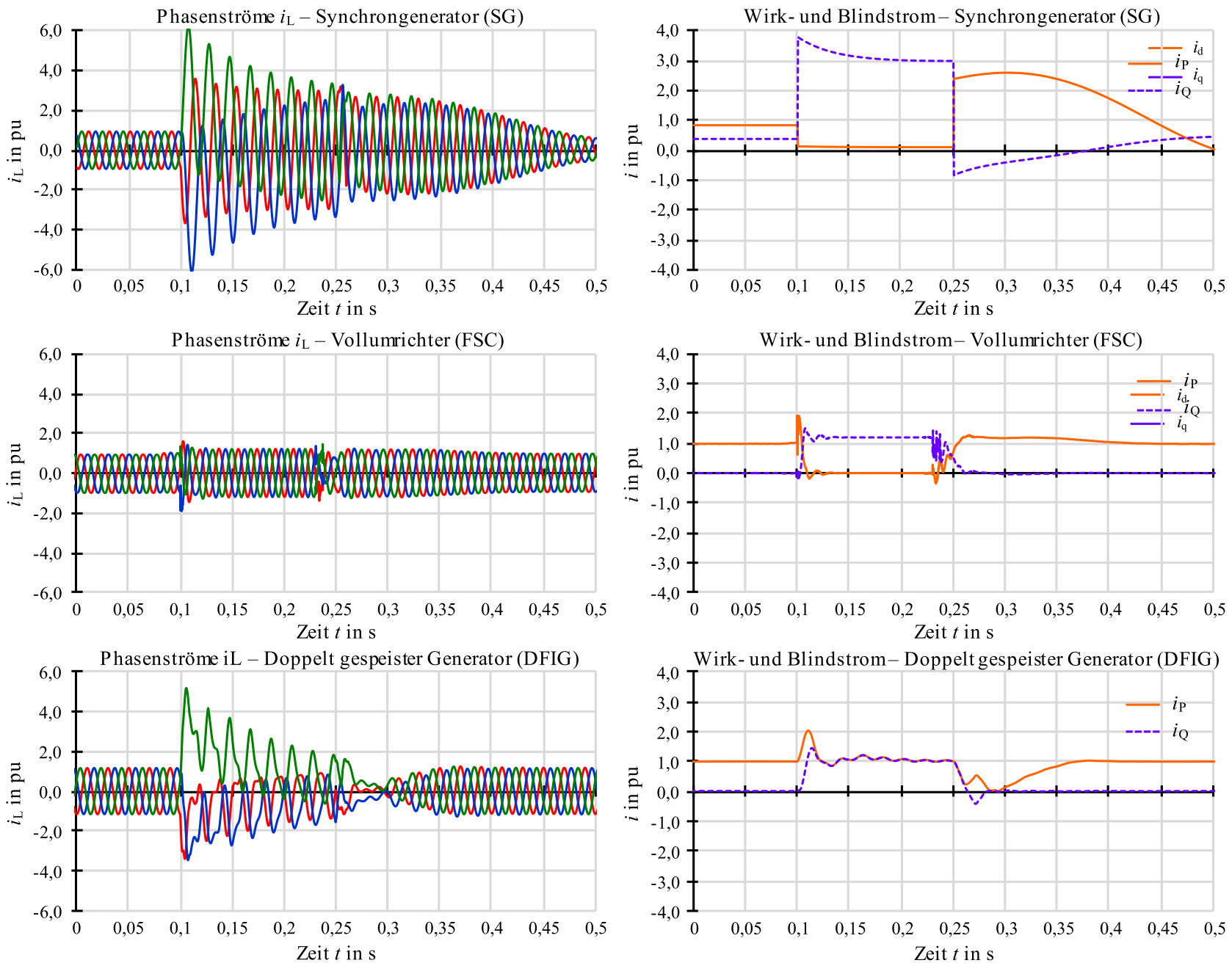

Abb. 2. Typische Kurzschlussstromverläufe von Synchronmaschinen (oben), WKW mit Vollumrichtern (Mitte) und WKW mit DFIG (unten). Bei der Synchronmaschine (Bemessungsleistung $90 \mathrm{MVA}$ ) wird von einem dreipoligen impedanzlosen Kurzschluss von 150 ms Dauer auf der Hochspannungsseite des Blocktransformators ausgegangen. Bei den WKW (Bemessungsleistung 2 MVA) ist der Kurzschluss ebenfalls auf der Hochspannungsseite angenommen. Die Kurven links zeigen die Leiterströme, rechts sind der Wirk- und Blindstrom im Mitsystem dargestellt. Bezugswert ist der Bemessungsstrom des Synchrongenerators bzw. des WKW. Für alle Simulation wurde ein detailliertes EMT (electromagnetic transient) Modell verwendet, welches auch sämtliche elektrischen Regelkreise (Umrichterregelung inklusive Phasenregelkreis, Erregung) sowie die Schutzeinrichtungen der Windkraftanlage (Crowbar, Chopper) beinhaltet

nungsverfahren dar. Es ermöglicht bestimmte Größen im Kurzschlussstromverlauf, die sog. charakteristischen Kurzschlussstromgrößen, mit einem Minimum an Daten (vorzugsweise Bemessungsdaten) und ohne Kenntnis des aktuellen Betriebszustandes vor Kurzschluss, d. h. ohne Lastflussberechnung, zu berechnen. Diese charakteristischen Kurzschlussstromgrößen sind der Stoßkurzschlussstrom, der Ausschaltwechselstrom, der Dauerkurzschlussstrom und der thermisch gleichwertige Kurzschlussstrom. Sie sind für die thermische und mechanische Festigkeit der Betriebsmittel und Anlagen maßgebend. Ihre Berechnung erfolgt mittels der Faktoren $\kappa, \mu, \lambda, m$ und $n$ aus dem Anfangs-Kurzschlusswechselstrom. Insofern kommt dem Anfangs-Kurzschlusswechselstrom eine zentrale Bedeutung zu.

Der Anfang-Kurzschlusswechselstrom $l_{k}^{\prime \prime}$ ist der Effektivwert des Wechselanteils im Kurzschlussstrom unmittelbar nach Kurzschlusseintritt. Er ist nicht messbar, lässt sich aber aus den inneren Spannungen bei den aktiven Betriebsmitteln Generatoren, Motoren, Netzeinspeisungen) sowie den Impedanzen der sonstigen Betriebsmittel exakt berechnen. Für den dreipoligen Kurzschluss gilt nach $[4,5]$ mit $U_{n}$ als der Netznennspannung an der Kurzschlussstelle

$$
\underline{l}_{k}^{\prime \prime}=\frac{c U_{n}}{\sqrt{3} \underline{z}_{k}}
$$

In GI. (2.1) ist $c U_{n} / \sqrt{3}$ die sog. Ersatzspannung und $\underline{Z}_{k}$ die resultierende Mitsystem-Kurzschlussimpedanz (Netzinnenimpedanz) jeweils an der Kurzschlussstelle. Die Ersatzspannung steht stellvertretend für die inneren Spannungen der Generatoren und Motoren. Die für den Kurzschluss von rotierenden Maschinen maßgebenden Impedanzen der Betriebsmittel werden gewöhnlich aus Datenblättern oder den Ersatzschaltplänen ermittelt. Diese sind oft frei zugängig oder sind vom Hersteller zu beziehen. Notfalls kann auf Richtwerte zurückgegriffen werden

Die Mitimpedanz auf der Oberspannungsseite (HV) des WKWTransformators (BT) soll nach der Gl. (28, IEC) berechnet werden:

$$
Z_{W D}=\frac{\sqrt{2} \kappa_{W D} U_{\text {rTHV }}}{\sqrt{3} i_{W D \text { max }}}
$$



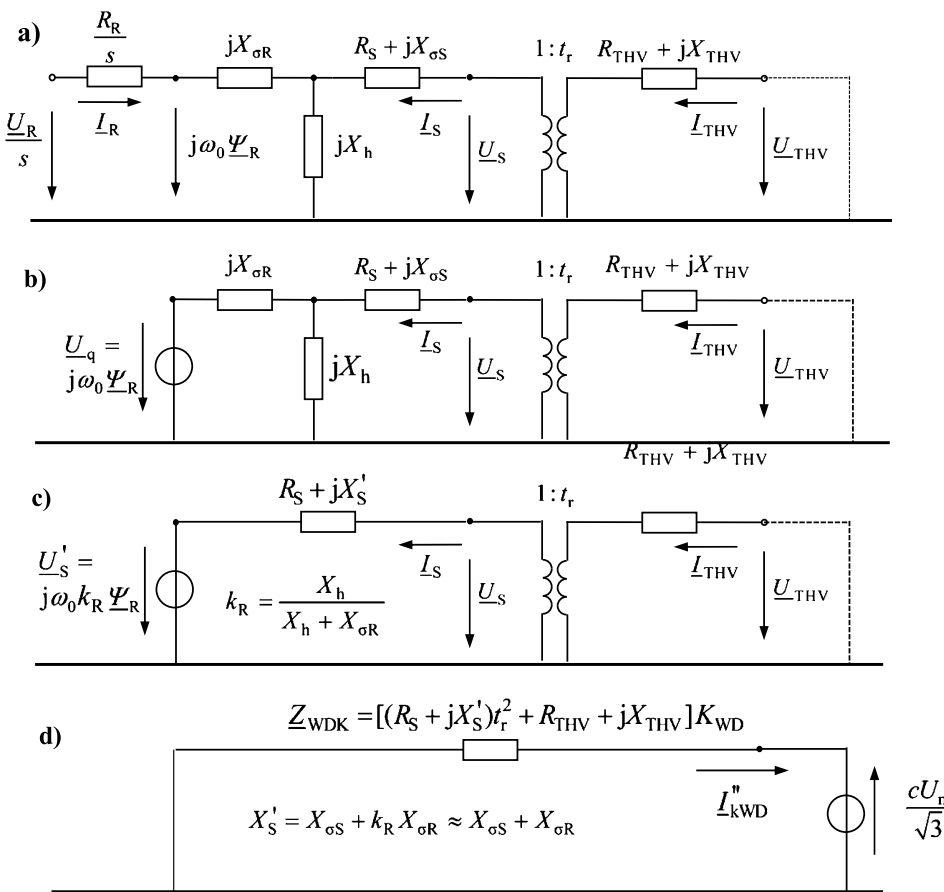

Abb. 3. Ersatzschaltungen zur Berechnung des dreipoligen Anfangs-Kurzschlusswechselstromes, (a) vollständig [6], (b) Einführung einer Spannungsquelle für die konstante Läuferflussverkettung, (c) Einführung der transienten Generatorspannung $\underline{U}_{S}^{\prime}$ und transienten Generatorreaktanz $X_{S}^{\prime}$ durch Umformen von Abb. 3b, (d) Einführung der Ersatzspannung an der Kurzschlussstelle

wobei i WDmax der höchstmögliche Momentanwert der Kurzschlussströme in einem der drei Leiter und $U_{\text {rTHV }}$ die Bemessungsspannung auf der Oberspannungsseite des WKW-Transformators ist. Er kann aus Kurzschlussversuchen oder dem Fault-Ride-Through (FRT)-Test $[7,8]$ bei der Zertifizierung der WEA ermittelt werden.

Der Faktor $\kappa$ wD kann dagegen im Kurzschlussversuch nicht ermittelt werden. Nach den Normen „muss” deshalb „wenn nicht bekannt" $\kappa$ WD $=1,7$ verwendet werden.

Der größte Maximalwert iwDmax tritt zufällig auf und sollte deshalb aus dem Raumzeiger der drei Leiterströme ermittelt werden. Es muss auch gewährleistet sein, dass der Kurzschluss im Bemessungsbetrieb erfolgt. Abweichende Versuchsbedingungen und unzutreffende Werte für $\kappa$ WD können zu Fehlern im AnfangsKurzschlusswechselstrom und den charakteristischen Kurzschlussstromgrößen führen. Nachteilig ist auch, dass iWDmax unter Umständen gar nicht zur Verfügung steht.

In dem zuständigen Normengremium UK 121.1 bei der DKE wird deshalb gegenwärtig diskutiert, die Kurzschlussimpedanz auf dem herkömmlichen Weg aus den Impedanzen der Ersatzschaltpläne für den Generator, den WKW-Transformator sowie der Kabelverbindung dazwischen zu ermitteln. Das hätte auch den Vorteil, dass man nicht nur den Betrag, sondern auch die komplexe Impedanz erhält. Diese Diskussion ist noch nicht finalisiert und wird hier vorab als Skizze vorgestellt, sie ist aktuell Gegenstand weiterer Untersuchungen und soll auch noch weiter mit Messergebnissen abgesichert werden.

Die Mitsystem-Ersatzschaltpläne für den doppelt speisenden Generator und den WKW- Transformator enthält Abb. 3. Die Impedanz der Kabel zwischen Generator und dem Transformator wird der Generator- oder Transformatorimpedanz zugeschlagen. Das Querglied des Transformators wird vernachlässigt.
Aus Abb. 3c folgt für die Kurzschlussimpedanz auf der OS-Seite des WKW-Transformators:

$$
\underline{Z}_{\mathrm{WD}}=t_{\mathrm{r}}^{2} \underline{Z}_{\mathrm{G}}+\underline{Z}_{\mathrm{THV}}=t_{\mathrm{r}}^{2}\left(R_{\mathrm{S}}+\mathrm{j} X_{\mathrm{S}}^{\prime}\right)+R_{\mathrm{THV}}+\mathrm{j} X_{\mathrm{THV}}=R_{\mathrm{WD}}+\mathrm{j} X_{\mathrm{WD}}
$$

und für den Anfangs-Kurzschlusswechselstrom ausgehend von $\underline{U}_{S}^{\prime}$ in der reellen Achse:

$$
\underline{\mathrm{l}}_{\mathrm{kWD}}^{\prime \prime}=\frac{t_{\mathrm{r}} U_{\mathrm{S}}^{\prime}}{\underline{Z}_{\mathrm{WD}}}
$$

Die transiente Generatorspannung $U_{S}^{\prime}$ ist der Rotorflussverkettung $\Psi_{R}$ proportional und bleibt wie diese beim Übergang vom stationären zum Kurzschluss-Zustand konstant. Um die Ersatzspannung an der Kurzschlussstelle einzuführen (Abb. 3d) wird die Gl. (2.3) im Zähler und Nenner mit dieser erweitert:

$$
\begin{aligned}
l_{-k W D}^{\prime \prime} & =\frac{t_{r} U_{S}^{\prime}}{\underline{Z}_{W D}} \cdot \frac{c U_{n} / \sqrt{3}}{c U_{n} / \sqrt{3}}=\frac{c U_{n}}{\sqrt{3} \underline{Z}_{W D}} \cdot \frac{t_{r} \sqrt{3} U_{S}^{\prime}}{c U_{n}} \\
& =\frac{c U_{n}}{\sqrt{3} \underline{Z}_{W D}} \cdot \frac{1}{K_{W D}}=\frac{c U_{n}}{\sqrt{3} \underline{Z}_{W D K}}
\end{aligned}
$$

Der Faktor $K_{W D}$ in Gl. (2.4) wird der Impedanz $\underline{Z}_{\text {WD }}$ zugeordnet und deshalb Impedanzkorrekturfaktor genannt. Er berechnet sich unter der Annahme, dass der Kurzschluss ausgehend vom Bemessungsbetrieb des Generators erfolgt, näherungsweise aus:

$$
K_{\mathrm{WD}}=\frac{c U_{\mathrm{n}}}{t_{\mathrm{r}} U_{\mathrm{rG}}\left(1+x_{\mathrm{S}}^{\prime} \sin \varphi_{\mathrm{rG}}\right)} \quad \text { mit } \quad x_{\mathrm{S}}^{\prime}=X_{\mathrm{S}}^{\prime} \frac{\sqrt{3} /_{\mathrm{rG}}}{U_{\mathrm{rG}}}
$$

Für die Gegensystemimpedanz gilt nach $[4,5] \underline{Z}_{2 \mathrm{WD}}=\underline{Z}_{\mathrm{WD}}$. Die Nullimpedanz $\underline{Z}_{0 W D}$ hängt von der Schaltgruppe des WKWTransformators und ggf. von dessen Art der Sternpunkterdung ab.

Der Stoßkurzschlussstrom ist der größtmögliche Momentanwert im Kurzschlussstromverlauf. Er ergibt sich aus der Summe des 


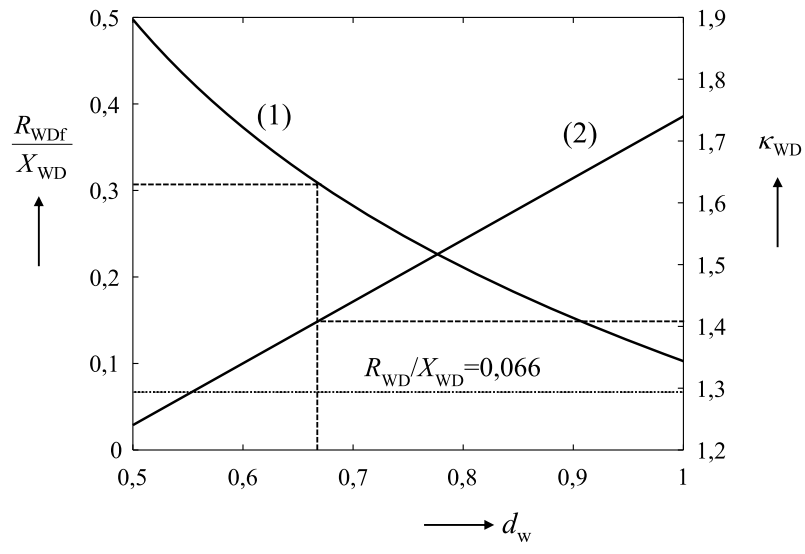

Abb. 4. Fiktives Verhältnis $R_{\text {WDf }} / X_{\text {WD }}$ (1) und Faktor $\kappa_{\text {WD }}$ (2) in Abhängigkeit vom Abklingfaktor des Wechselanteils für eine 4,5-MW-WKW mit DFIG (berechnet nach GI. (2.6) und GI. (57, IEC)

Wechsel- und Gleichanteils im Kurzschlussstrom zum Zeitpunkt $t_{\mathrm{p}}$ seines Auftretens:

$$
\begin{aligned}
i_{\mathrm{pWD}} & =i_{\mathrm{kWWD}}\left(t_{\mathrm{p}}\right)+i_{\mathrm{kgWD}}\left(t_{\mathrm{p}}\right)=\sqrt{2} l_{\mathrm{kWD}}^{\prime \prime}\left[d_{\mathrm{W}}\left(t_{\mathrm{p}}\right)+\frac{l_{\mathrm{gWD}}}{\sqrt{2} l_{\mathrm{kWD}}^{\prime \prime}} \mathrm{e}^{-\frac{t_{\mathrm{p}}}{\mathrm{T}_{\mathrm{gWD}}}}\right] \\
& =\sqrt{2} l_{\mathrm{kWD}}^{\prime \prime} \kappa \mathrm{WD}
\end{aligned}
$$

Der Faktor $d_{w}\left(t_{p}\right)$ steht für das Abklingen des Wechselanteils und die Anteile des Umrichters, IgWD ist der Anfangswert des Gleichgliedes und $T_{\mathrm{gWD}}=X_{\mathrm{WD}} /\left(\omega_{0} R_{\mathrm{WD}}\right)$ die Gleichstromzeitkonstante. Der Anfangs-Kurzschlusswechselstrom und der Gleichanteil des vom Generator gelieferten Stroms sind unabhängig vom Verhalten des Umrichters.

Für den (Stoß)Faktor $\kappa$ WD soll der Ausdruck $\kappa=1,02+0,98 \mathrm{e}^{-3 R / X}$ nach GI. (57, IEC) verwendet werden. Dieser berücksichtigt jedoch nicht das Abklingen des Wechselanteils und nicht die Umrichteranteile und gilt auch nur für $I_{g W D} \approx \sqrt{2} l_{k W D}^{\prime \prime}$ und $t_{p}=9,55 \mathrm{~ms}$. Durch das Abklingen des Wechselanteils und dessen höherer Frequenz tritt der Stoßkurzschlussstrom bei den DFIG zu einem früheren Zeitpunkt auf. Um die GI. (57, IEC) dennoch anzuwenden, muss deshalb von einem fiktiven Verhältnis $R_{\text {WDf }} / X_{\text {WD }}>R_{\text {WD }} / X_{\text {WD }}$ ausgegangen werden. Davon wird auch jetzt schon in den Normen für die Synchrongeneratoren Gebrauch gemacht.

Zur Bestimmung repräsentativer Werte für $R_{\mathrm{WDf}} / X_{\mathrm{WD}}$ sind Kurzschlussversuche auszuwerten. Aus dem daraus bestimmten maximal möglichen Kurzschlussstrom (Stoßkurzschlussstrom) und dem unter Versuchsbedingungen nach Gl. (2.3) berechneten AnfangsKurzschlusswechselstrom erhält man:

$$
\frac{R_{\mathrm{WDf}}}{X_{\mathrm{WD}}}=-\frac{1}{3} \ln \left[\frac{1}{0,98}\left(\frac{i_{\mathrm{kWDmax}}}{\sqrt{2} l_{\mathrm{kWD}}^{\prime \prime}}-1,02\right)\right] ; \quad \frac{i_{\mathrm{kWDmax}}}{\sqrt{2} l_{\mathrm{kWD}}^{\prime \prime}}>1,02
$$

Das Verhältnis $R_{\text {WDf }} / X_{\text {WD }}$ ist umso größer, desto stärker der Wechselanteil abklingt (Abb. 4). Die Auswertung von Kurzschlussversuchen an einem 4,5-MW-WKW haben für den Abklingfaktor des Wechselanteils ein Mittelwert von $d_{w}=0,67$ ergeben. Dem entspricht ein fiktives Verhältnis $R_{W D f} / X_{W D}=0,305$ und ein Faktor $\kappa \mathrm{WD}=1,41$. Das natürliche Verhältnis $R_{\mathrm{WD}} / X_{\mathrm{WD}}$ beträgt dagegen nur 0,066 und ergibt einen Faktor $\kappa=1,82$.

Für den Ausschaltwechselstrom und den Dauerkurzschlussstrom bei symmetrischen Kurzschlüssen kann angenommen werden, dass der Wechselanteil auf seinen stationären Endwert abgeklungen ist. Dieser hängt vom Regelkonzept und den Anforderungen zur Blindstromeinspeisung des jeweiligen Netzbetreibers bzw. standortabhängigen Anforderungen $a b$ und liegt in der Regel bei $1,1 \cdot I_{\mathrm{rWD}}$, teilweise aber auch bis $(1,3 \ldots 1,5) \cdot I_{r W D}$. Die minimal geforderte Dauer der Stromeinspeisung ist in der Regel durch den Netzbetreiber vorgegeben, die maximale Dauer hängt vom Regelkonzept und Einstellungen des Netz- und Anlagenschutzes abhängen, ihre Berücksichtigung ist jedoch nicht Gegenstand der Beschreibung in der Norm.

\section{Stationäres Verhalten der DFIG im Normalbetrieb}

Für eine Analyse des Verhaltens der Anlage im Normalbetrieb wird zunächst ein typisches Blockschaltbild und Regelschema einer solchen Anlage gemäß Abb. 5 betrachtet. Bei niedrigen Windgeschwindigkeiten wird die Drehzahl durch die WKW-Steuerung mit Hilfe eines Sollwerts der Wirkleistungsabgabe für den rotor- oder netzseitigen Umrichter eingeregelt. Der jeweils andere Umrichter regelt dann die Zwischenkreisspannung ein. Überschreitet die Windgeschwindigkeit den für die Einspeisung von Bemessungsleistung notwendigen Wert, erfolgt eine Begrenzung der Leistungsaufnahme durch ein Verstellen der Rotorblätter. An den Umrichter wird dann eine konstante Leistung oder ein konstantes Moment vorgegeben.

Die Regelung der Blindleistungsabgabe der WKW erfolgt durch eine Kombination der Einspeisung über den Stator mit Hilfe des rotorseitigen Umrichters und über den netzseitigen Umrichter. Früher wurde eher ein Leistungsfaktor am Netzanschlusspunkt (NAP) des Windparks eingeregelt, inzwischen erfolgt meist die Vorgabe einer $Q(U)$-Statik am NAP (z. B. [9-12]). Die Maschine wird daher im übererregten Bereich in der Regel komplett von der Rotorseite her magnetisiert. Je nach Konzept regelt der netzseitige Umrichter oder der maschinenseitige Umrichter die Zwischenkreisspannung. Ein weiterer Bestandteil des elektrischen Regelschemas ist ein Phasenregelkreis (phase locked loop, PLL), welcher benötigt wird, um Phase des Statorspannungszeigers als Referenzwinkel für die Umrichterregelung zu erhalten.

Im Normalbetrieb, d. h. wenn es kein Leistungs- oder Spannungsgrenzwert erreicht ist, verhalten sich daher Synchronmaschine, FSC und DFIG am Netz sehr ähnlich. Durch die Möglichkeit der schnellen Leistungseinstellung durch den Umrichter ist jedoch bei den umrichtergekoppelten Anlagen, wenn gewünscht, eine deutliche schnellere Arbeitspunktanpassung möglich. Welchen Beitrag umrichtergekoppelte Anlagen zur Trägheit und Kurzschlussleistung im stationären Betrieb leisten, ist stark von der implementierten Umrichterregelung abhängig.

\section{Verhalten der DFIG im Fehlerfall}

Wesentlich für das Verhalten der DFIG Anlagen im Fehlerfall ist die Forderung der sog. dynamischen Netzstützung (angelehnt an das Verhalten von Synchronmaschinen bei Spannungsänderung), wie sie in den Netzanschlussbedingungen ([9-11]) festgelegt sind. Die Anlagen müssen dabei wie alle nichtsynchronen Stromerzeugungsanlagen im Falle einer sprunghaften Spannungsänderung $|\Delta u|>0,1$ p.u. die Spannung durch einen zusätzlichen Blindstrom $\Delta i_{B}$ im Mitsystem (Index "1") und Gegensystem (Index "2") stützen, es gilt dabei $\Delta i_{\mathrm{B} 1,2}=k \cdot \Delta u_{1,2}$, mit einem vom Netzbetreiber definierten Proportionalitätsfaktor $k$, wobei $2 \leq k \leq 6$. Dieser Blindstrom wird analog zum Verhalten von Synchrongeneratoren - zusätzlich zu dem für die stationäre Spannungshaltung geliefertem Blindstrom eingespeist und hat Vorrang gegenüber einer Wirkstromeinspeisung, welche gegebenenfalls gegenüber dem Blindstrom zu reduzieren ist. Um einen Blindstrom definieren zu können, ist stets ein Spannungszeiger notwendig, für diesen wird meist die Spannung an der Unterspannungsseite des WKW-Transformators herangezogen.

Die Asynchronmaschine verhält sich bei Kurzschlusseintritt zunächst ähnlich einer Synchronmaschine und liefert bei einem Einbruch der Netzspannung zunächst einen hohen Blindstrom, mit dem 


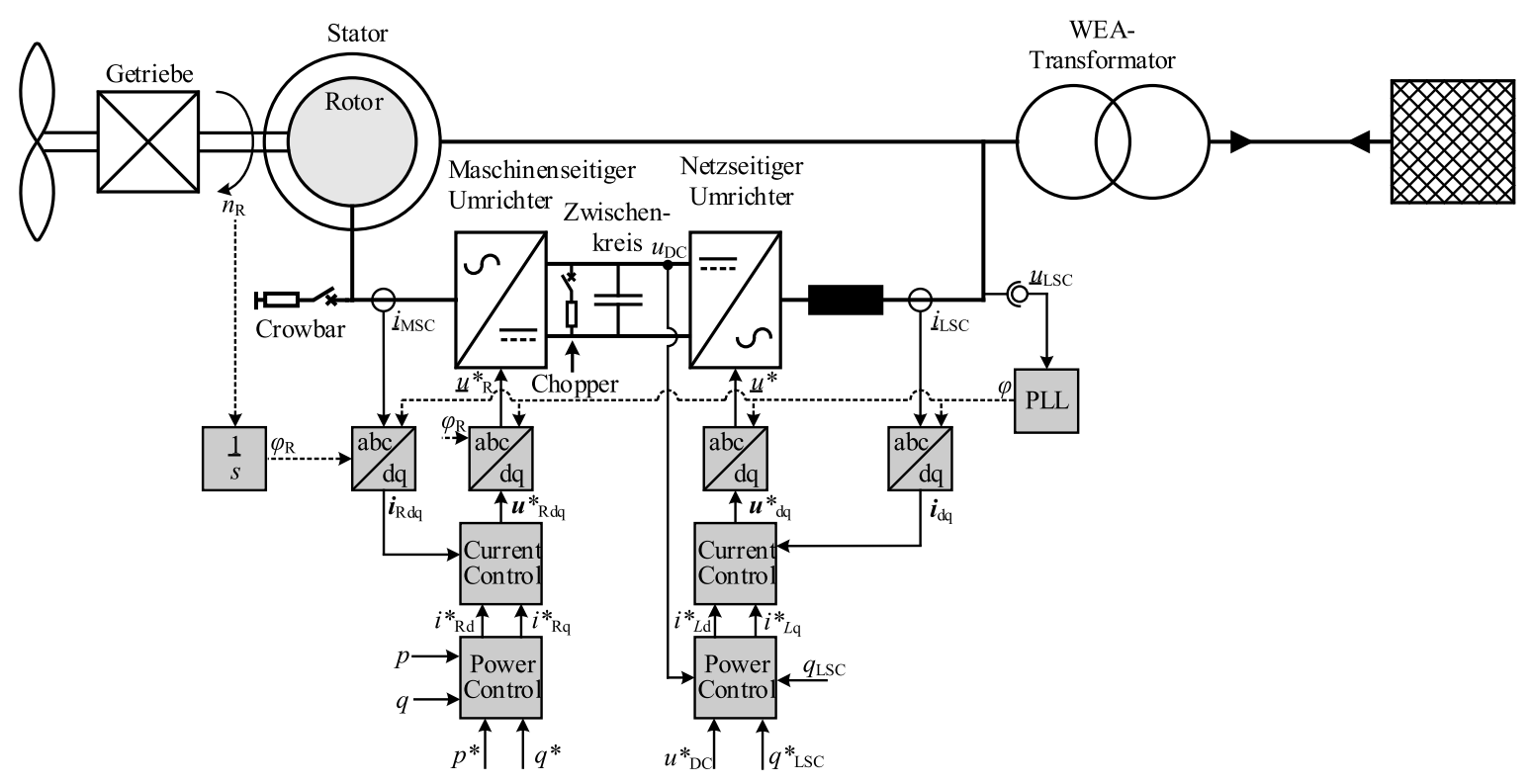

Abb. 5. Vereinfachtes Übersichtsschaltbild einer DFIG mit den wichtigsten Regelkreisen und Schutzeinrichtungen. Der WKW-Transformator ist oft auch als Dreiwickler ausgeführt, er ist über eine (hier nicht explizit dargestellte) Kabelverbindung mit der Maschine und dem Umrichter verbunden

auch ein hoher Rotorstrom verbunden ist. Entscheidend für die Auslegung des Umrichters ist es, diese anfänglich sehr hohen Ströme zu beherrschen, ohne dass es zu einer Überlastung der aktiven Schalter (IGBTs) oder zu einer Spannungsüberhöhung im Zwischenkreis kommt.

Dies ist notwendig um die Anforderungen der Regeldynamik aus den Anschlussbedingungen zu erfüllen, so ist z. B. in [9] eine Anschwingzeit $T_{90 \%}$ von $\leq 30$ ms und eine Einschwingzeit von $\leq 60 \mathrm{~ms}$ gefordert. Diese Werte sind jeweils bezogen auf den nach [7] gemessenen Mitsystemwert, der sich wie ein gleitender 20 msMittelwertfilter auswirkt - die tatsächliche Stromantwort muss damit deutlich schneller erfolgen.

Beim Einsatz eines Choppers im Zwischenkreis ist die Rotorspannung im Falle eines Kurzschlusses beim Überschreiten der Stromgrenzen der IGBTs über die nun als Diodenbrücke funktionierenden IGBTs auf die Zwischenkreisspannung gedeckelt. Ziel der Regelung des maschinenseitigen Umrichters ist es, mit der Stromregelung schnell wieder aufzusetzen, sobald der Strom in der jeweiligen Phase unter den Grenzwert des IGBT-Strom sinkt und so zu vermeiden, dass es zu weiteren Pulssperren kommt, z. B. durch eine Reduktion der Wirkstromabgabe des Stators.

Der netzseitige Umrichter kann - in Ergänzung zum Stator - zusätzlichen Blindstrom einspeisen. Der Gleichanteil des Ständerstroms führt zu einem mit der Rotordrehzahl transformierten Stromanteil, der durch den maschinenseitigen Umrichter nicht direkt beeinflussbar ist. Mit Hilfe des netzseitigen Umrichters kann aber der am Anschlusspunkt der WKW ins Netz fließende Strom besser geregelt werden, damit der Blindstrom im Mitsystem auf dem Sollwert gehalten werden kann.

Für die Dimensionierung der DFIG ist vor allem das Verhalten bei unsymmetrischen Fehlern entscheidend, da hier das auftretende Gegensystem beherrscht werden muss. Das Verhalten von DFIGAnlagen im Falle von unsymmetrischen Fehlern ist komplex und stark von der implementierten Regelungsstrategie abhängig, im Rahmen dieser Arbeit wird darauf jedoch nicht näher eingegangen.

\section{Aspekte des Anlagen- und Netzschutzes}

\subsection{Anlagenschutz}

Zunächst soll ein kurzer Überblick auf den internen Schutz des Umrichters der DFIG gegeben werden. Er verhindert bei Fehlern eine Zerstörung der dort eingesetzten Komponenten, welche im Gegensatz zum relativ robusten Generator kaum überlastfähig sind. Konkret ist besonders auf die Einhaltung der Stromgrenzwerte der Halbleiterventile sowie auf die Spannungsgrenze des Zwischenkreiskondensators zu achten.

Es gibt gemäß [3] zwei zu betrachtende Varianten der DFIG, Typ 3a mit "Zwischenkreischopper", und Typ 3b mit geregelter "Crowbar". Im Fall 3a erfolgt im Fehlerfall (bei Kurzschluss) - sofern die Stromtragfähigkeit der IGBTs im aktiven Betrieb erreicht wird - eine Pulssperre der IGBTs und ein Stromfluss über die Freilaufdioden der IGBT-Brücke des maschinenseitigen Umrichters in den Zwischenkreis. Die Energie wird dann über einen getakteten Widerstand ("Chopper") im Zwischenkreis in Wärme umgesetzt, so dass der Betrieb des netzseitigen Umrichters nicht beeinträchtigt wird.

Im Fall 3b erfolgt bei Überschreiten der Stromgrenzen der IGBTs ein Kurzschließen des Rotorkreises (je nach Auslegung mit oder ohne Widerstände) mit Hilfe von Thyristoren. Dies ermöglicht eine kleinere Auslegung des maschinenseitigen Umrichters, allerdings können damit die aktuellen EU-Anforderungen an die Blindstromeinspeisung im Fehlerfall nicht erfüllt werden, da der kurzgeschlossene Asynchrongenerator nach Entmagnetisierung beim Spannungseinbruch induktiv wird.

\subsection{Netzschutz}

Im Sinne einer bestmöglichen Betriebsführung, auch im Störungsfall, stellt die Einhaltung des Sachgüter- und Personenschutzes von Anlagen und ganzen Netzen eine wesentliche Voraussetzung für deren zulässigen und sicheren Betrieb dar, wobei es im Falle von DFIG besondere Anforderungen gibt, welche sich von Schutzkonzepten von Netzen, welche von Synchrongeneratoren oder Vollumrichtern (FSC) gespeist werden, deutlich unterscheiden. 


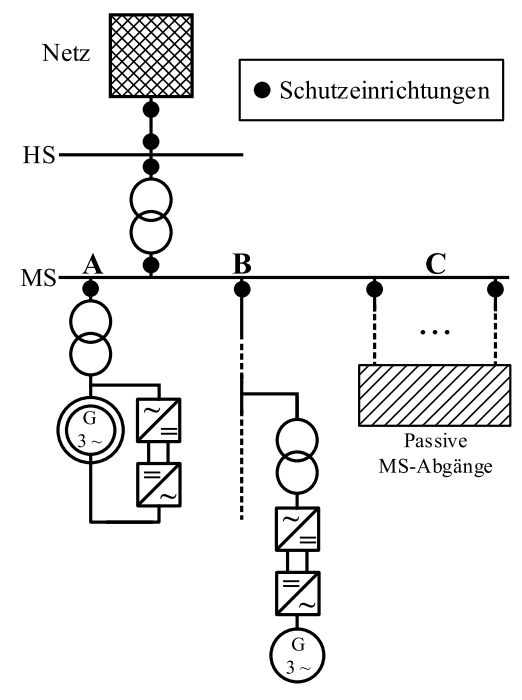

Abb. 6. Betrachtete Schutzkonfiguration mit Windkraftanlagen, exemplarisch dargestellt durch eine DFIG Anlage und eine Vollumrichteranlage sowie weiteren Abgängen auf der MS-Ebene

Die nachfolgenden Ausführungen beziehen sich demgemäß vorrangig auf den Anlagen- und Schutz von Netzen mit relevanter DFIG-Einspeisung, welcher die Resilienz der einzelnen Anlagen bzw. Netze hinsichtlich der Fehlerarten Kurzschluss und Unterfunktion von Schutzeinrichtungen erhöhen soll.

Gemäß der Methodik bei der technischen Auslegung von Schutzkonzepten sind nicht nur alle relevanten Fehlerarten hinsichtlich ihrer einwandfreien Klärung durch das Schutzsystem zu prüfen, sondern auch das Auftreten dieser Fehler an allen kritischen Fehlerorten für alle als berücksichtigenswert erachteten Betriebszustände des Netzes bzw. der Anlagen, u. U. auch in einem Inselbetrieb. Dieser kann im Sinne von "autarken Netzen" bewusst oder im Zuge eines Störungsgeschehens, zum Beispiel durch eine reserveschutzbedingte Abschaltung des HS/MS- Transformators, erfolgen.

In der Folge wird von einer Netzkonfiguration gemäß Abb. 6 ausgegangen. Die Erzeugungsanlagen sind musterhaft in der Mittelspannungs- (MS)-Ebene, direkt an der Sammelschiene (Abb. 6 / A) sowie im MS-Netz (Abb. 6 / B), angeschlossen. Die passiven MS-Abgänge sind in Abb. 6 mit C gekennzeichnet.

\subsection{Netzschutzeinrichtungen}

Üblicherweise werden die Schutzfunktionen gemäß folgender Systematik eingeteilt:

- Netzschutzeinrichtungen: Fehlerbehandlung im öffentlichen Netz (Netzschutz)

- Entkupplungsschutzeinrichtungen bei einer Windkraftanlage: Fehlerbehandlung im elektrischen Energiesystem (Systemschutz)

Je nachdem, ob Windparks elektrische Leistung in ein Hochspannungs- oder ein Mittelspannungsnetz einspeisen, sind deren Schutzeinrichtungen verschieden. In der Hochspannungsebene dominieren Distanzschutzeinrichtungen mit verschiedenen Zusatzfunktionen wie z.B. Unterimpedanzanregung, Signalvergleich mit Freigabe- bzw. Blockierverfahren, automatische Wiedereinschaltung, u. U. redundant ausgeführte Erdschlusschutzeinrichtungen, Synchrocheck, Kennlinienumschaltung. Zunehmend finden auch in der Hochspannungsebene Leitungs-Differenzialschutzeinrichtungen Verwendung. In den Mittelspannungsnetzen hingegen werden einfachere Schutzkonzepte, wie das Überstromzeitschutz-Verfahren

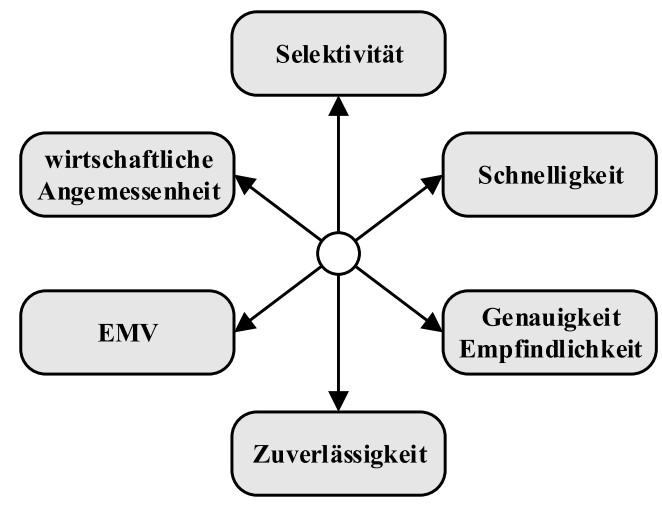

Abb. 7. Allgemeine Anforderungen an Schutzeinrichtungen [13]

Tab. 3. Einstellungen des Entkupplungsschutzes bei Anschluss der Erzeugungseinrichtung in der Mittelspannungsebene [9]

\begin{tabular}{lll}
\hline \multicolumn{2}{l}{ Schutzeinstellung - Sammelschiene } \\
\hline$U \gg$ & $1,25 \cdot U_{\mathrm{MS}}$ & $0,1 \mathrm{~s}$ \\
$U<$ & $0,80 \cdot U_{\mathrm{NS}}$ & $1,5 . .2,4 \mathrm{~s}$ (gestaffelt) \\
$U \ll$ & $0,8 \mathrm{~s}$ \\
\multicolumn{2}{l}{ Schutzeinstellung - im MS-Netz } \\
$U \gg$ & \\
$U<$ & $1,25 \cdot U_{\mathrm{MS}}$ & $0,1 \mathrm{~s}$ \\
$U \ll$ & $0,80 \cdot U_{\mathrm{NS}}$ & $1,0 \mathrm{~s}$ \\
\hline
\end{tabular}

und Distanzschutzeinrichtungen, oft nur mit Überstromanregung, eingesetzt.

Die Netze und Anlagenschutzeinrichtungen mit DFIG-Einspeisung müssen eine Vielfalt von Erfordernissen zu erfüllen, allen voran aber stehen Selektivität und Zuverlässigkeit (siehe Abb. 7).

Da Netzschutzeinrichtungen, wie andere Schutzeinrichtungen auch, im Allgemeinen nach dem Prinzip „Hauptschutz mit integrierter Reserveschutzfunktion" und damit als Unit Protection ausgelegt sind, kommt den Schutzeinrichtungen von DFIG-Einspeisungen in diesem Zusammenhang die Sicherstellung der Reserveschutzfunktion zu. Da sowohl in der Mittel- als auch in der Hochspannungsebene der Reserveschutz nach dem Zeitstaffelprinzip und damit im Zeitfenster von $1 \mathrm{~s}$ und aufwärts arbeitet, ist die Sicherstellung eines ausreichenden Stromflusses oder entsprechender Ersatzmaßnahmen unter Umständen eine kritische Aufgabe: Bekanntermaßen muss beim Zeitstaffelschutz für den Fall, dass eine Schutzeinrichtung ihre Aufgabe nicht erfüllt, für die Reservestufe noch ausreichend einschließlich der Sicherheitsmargen von z. B. $100 \%$-Strom bis zu deren Auslösezeit sichergestellt sein.

\subsection{Kurzschlussschutzeinrichtungen des Anschlussnehmers} Als Hauptschutz übernehmen die Kurzschlussschutzeinrichtungen der DFIG-Windkraftanlage einschließlich der Reservefunktion im Falle einer nicht erfolgreichen Fehlerabschaltung in dieser Anlage.

Im Sinne eines Reserveschutzes allerdings muss das Kurzschlussschutzkonzept der WKW an das Hauptschutzkonzept des jeweils entsprechenden Mittelspannungs- bzw. Hochspannungsnetzes angepasst werden. Wegen der höheren Auslösezeiten im Reserveschutzfall ist wiederum unter Umständen die Sicherstellung eines ausreichenden Stromflusses herausfordernd. 


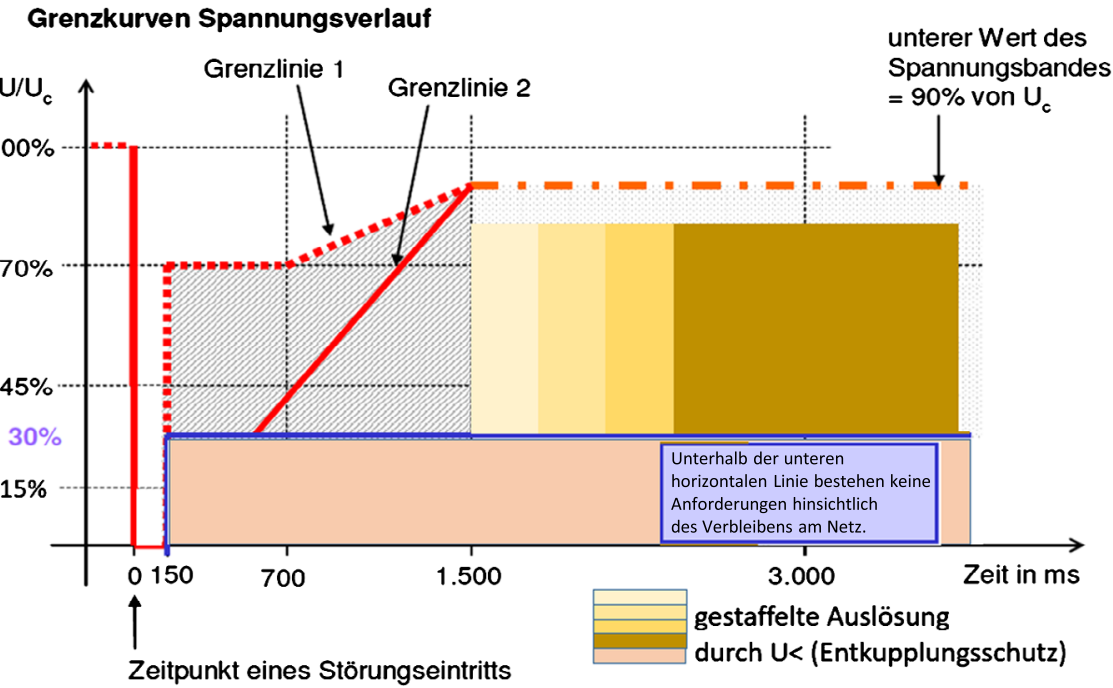

Abb. 8. Grenzlinien für den Spannungsverlauf am Netzanschlusspunkt für eine Erzeugungsanlage vom Typ 2 (kein Synchrongenerator) [9]

\subsection{Entkupplungsschutzeinrichtungen bei Erzeugungsanlagen}

Entkupplungseinrichtungen greifen als Teil des Systemschutzes in den Netzbetrieb dann ein, wenn bei stark gestörten Betriebszuständen des Netzes eine klare Abtrennung der dezentralen Erzeugungsanlagen sowohl für diese selbst als auch für das Netz die relativ beste Strategie zur Störungsbehandlung ist. Den einschlägigen Vorschriften sind implizit Spannungsquellen zugrunde gelegt, deren Output im Störungsfall nicht zeitabhängig, sondern konstant ist. Diese Voraussetzung ist allerdings bei DFIG-Anlagen nicht erfüllt. An dieser Stelle werden deshalb die Kernpunkte des derzeitig gültigen Regelwerks exkursorisch wiedergegeben.

Da der Entkupplungsschutz als Systemschutz arbeitet und damit nicht mehr betriebsmittelselektiv ist, werden hier Spannung und Frequenz als globale Netzparameter für die Einstellung herangezogen. Typische Einstellwerte werden in der folgenden Tabelle 3 wiedergegeben:

Eine Netzentkupplung, d. h. die Trennung von Erzeugungsleistung, darf aber auf keinen Fall zu früh erfolgen, um ein Absinken der Systemfrequenz nach Fehlerklärung auf ein Minimum zu begrenzen. Abhängig vom Anschlusspunkt (MS, HS, HöS) werden unterschiedliche Grenzkurven für die Spannung in den Netzanschlussregeln vorgegeben, oberhalb derer keine Netztrennung erfolgen darf. In Abb. 8 ist eine solche Grenzkurve für die Mittelspannungsebene beispielhaft wiedergegeben (aus [9]), des Weiteren ist auch der Wirksamkeitsbereich des Unterspannungsschutzes eingetragen.

Bemerkung: Da empfohlen wird, je Hochspannungsnetz jeweils ein Viertel der Erzeugungsanlagen im Zeitraum von 1,5 s bis 2,4 $s$ vom Netz zu trennen, erfolgt deren Trennung bei einer Netzentkupplung gestaffelt.

In Netzen mit überwiegender DFIG-Einspeisung ist die längerzeitige Spannungskonstanz wegen der rasch abklingenden Stromeinspeisung seitens der Quelle im Fehlerfall nicht mehr gegeben (siehe Abb. 2). Daraus resultiert, dass im Sekundenbereich die Netzspannung durchaus unterhalb von $30 \%$ liegen kann und damit der Arbeitspunkt des Netzes in denjenigen Bereich kommt, für den keine Anforderung hinsichtlich des Verbleibens im Netz mehr bestehen.

Trotzdem sind im Sinne der Aufrechterhaltung definierter Netzzustände Schutzfunktionen gefordert, und diese beziehen ihre Signale aus den Fehlerströmen.

\subsection{Auswirkung des abnehmenden Kurzschlussstroms von} DFIG-Einspeisungen für strombasierte Schutzfunktionen Im Gegensatz zum Anfangskurzschlussstrom bei einem dreipoligen Klemmenkurzschluss, der vor allem durch die Induktivität von Generator, Kabeln und Transformator bestimmt ist, und der im Sinne einer groben Abschätzung oft mit dem 6- bis 8-fachen des Bemessungsstroms einer DFIG angegeben wird, nimmt der - durch die Stromgrenzen des Umrichters limitierte - Dauerkurzschlussstrom rasch auf maximal den Bemessungsstrom ab. Im Fehlerfall wird der Strom auf das im Umrichter eingestellte Verhältnis von Wirk- und Blindstrom in Abhängigkeit von der Spannung eingeregelt. Der Gleichanteil des Statorflusses der Generators lässt sich mit dem Umrichter nicht direkt regeln und führt zu einem abklingenden drehzahlfrequenten Anteil, der sich auch mit der Kombination aus rotorseitiger Regelung und Netzumrichter in der Praxis nicht vollständig ausregeln lässt (siehe den Vergleich des Blindstroms von DFIG und Vollumrichter in Abb. 2).

Allerdings ist aus schutztechnischer Sicht dieser Strombeitrag einer DFIG unter Berücksichtigung der Forderung nach einem ausreichenden Ansprechpegel für jeden Fehlerort und in jeder Betriebsweise des Netzes, auch bei tief eingestellten Anregewerten, als nicht immer sichergestellt einzustufen.

Bei Worst-Case-Betrachtungen bedeutet das für hohe Abschaltzeiten, dass die Freigabewerte für allfällige Reserveschutzfunktionen nicht mehr in der Größenordnung der Nennströme der Betriebsmittel sind, sondern weiter darunterliegen. Wenn diesem Umstand Rechnung getragen wird, ist ein Schutzversagen bei ungünstigen Speiseverhältnissen oder im Inselbetrieb zu erwarten. Da die allgemeine Schutzphilosophie besagt, dass einem Zeitstaffelschutzverfahren die Erzeugungseinheiten als letzte vom Netz abgeschaltet werden, können sich für ungünstige Fälle hohe Abschaltzeiten ergeben.

\subsection{Erweiterte Schutzfunktionalität bei sehr kleinen Kurzschlussströmen}

Es gibt zwei Möglichkeiten, dem soeben aufgezeigten Dilemma des mittelfristig sehr kleinen und langfristig möglicherweise fehlenden Kurzschlussstromes seitens einer DFIG zu entkommen. Davon ist die bekannteste die (tief eingestellte) Überstromauslösung mit Unterspannungsfreigabe: Hierbei wird beim Unterschreiten einer Span- 


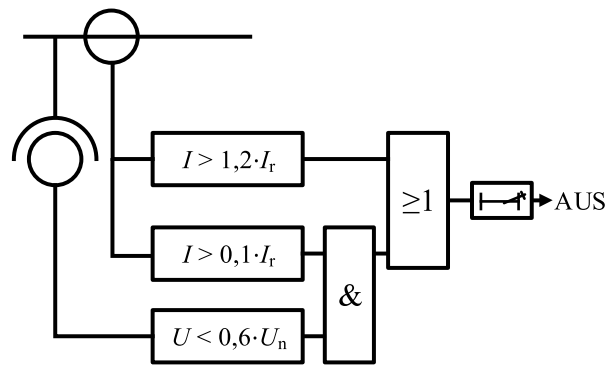

Abb. 9. Blockschaltbild für die unterspannungsgesteuerte Überstromauslösung

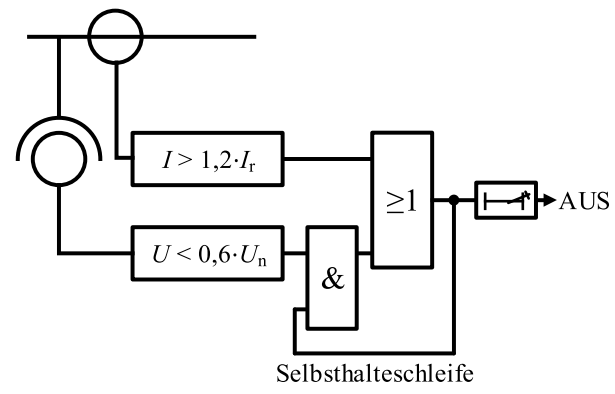

Abb. 10. Zeitstaffelschutz mittels unterspannungsgesteuerter Anrege-Selbsthaltung

nungsgrenze die Ansprechempfindlichkeit der Überstromschutzeinrichtungen stark herabgesetzt, um mit dem verbleibenden geringen Fehlerstrom noch eine Reserveschutzauslösung sicherzustellen. Typische Ansprechwerte (Größenordnung) sind für diese Art der Überstromanregung $I_{\text {Anregung }}=0,1 \cdot I_{r}$ und für die Unterspannungsfreigabe $U_{\text {Freigabe }}=0,6 \cdot U_{n}$.

Der ANSI-Code für eine solche Schutzeinrichtung lautet 51V (voltage restrained time overcurrent). In Abb. 9 ist das Blockschaltbild für dieses Prinzip vereinfacht dargestellt [14].

Bei diesem Verfahren bleibt die Grundfunktionalität des StromZeitschutzes erhalten, es wird aber zusätzlich - sobald die Spannung einen gewissen Wert unterschreitet, und solange noch ein gewisser Reststrom vorhanden ist, ein zweiter Auslösepfad aktiviert. Dieses Verfahren setzt allerdings voraus, dass nach der oft hohen Reserveschutzzeit (im Bereich einiger Sekunden) noch ausreichend Strom vorhanden ist. Eine weitere Schwachstelle des Verfahrens ist die Unterspannungsfreigabe. Wenn durch Manipulation oder Automatenfall die Messspannung gestört ist, erfolgt im normalen Lastbetrieb eine Auslösung.

\subsection{Erweiterte Schutzfunktionalität mittels}

unterspannungsgesteuerter Anrege-Selbsthaltung

Bei dieser Schutzfunktion wird durch den starken Anfangskurzschlussstrom einer DFIG, der entsprechend der Charakteristik der Maschine ein Vielfaches des Bemessungsstroms beträgt, in Verbindung mit dem Verfall der Spannung eine Selbsthalteschleife aktiviert. Nach einer eingestellten Zeit erfolgt die Auslösung. Falls aber in der Zwischenzeit durch eine andere Schutzeinrichtung das fehlerhafte Netzsegment aus dem Netzverbund herausgetrennt und unter der Annahme verbleibender, ausreichender Einspeisekapazitäten - die gesunde Netzspannung wiederhergestellt wurde, wird die Selbsthalteschleife unterbrochen, und die Erzeugungsanlage verbleibt im entsprechendem Netzverbund. Das Blockschaltbild dieser Funktion ist in Abb. 10 wiedergegeben.
Mit diesem Schutzverfahren wird der Forderung Rechenschaft getragen, bei Realisierung der Reserveschutzfusion auf Basis von Zeitstaffelung die Einspeisungen als letzte abzuschalten, auch wenn de facto kein ausreichender Kurzschlussstrom vorhanden ist.

\section{Zusammenfassung und Ausblick}

Die Bedeutung der Windkraftwerke für die Erzeugungsstruktur bedingt eine verlässliche Integration in Planung und Betrieb elektrischer Energienetze, dies umso mehr, als das das zukünftige Netz eine von leistungselektronisch gekoppelten Elementen dominierte Struktur aufweisen wird. Ein technischer Eckpfeiler dieser Integration ist das sichere Beherrschen des Verhaltens im Fehlerfall. In diesem Artikel wurden wichtige Aspekte des Verhaltens praktisch relevanter Elemente dieser Erzeugungsanlagen, den doppelt speisenden Generatoren, wie sie in Windkraftwerke sowie zunehmend auch in drehzahlvariablen Pumpspeicherkraftwerken Anwendung finden, aufgezeigt.

Deren Kurzschlussverhalten ist komplex und durch ein Zusammenspiel der Maschine mit dem Umrichter bestimmt. Das Verhalten des Umrichters hängt maßgeblich von der implementierten Regelstrategie ab. Beim Regelverhalten solcher Anlagen sind zukünftig Änderungen der Regelstruktur zu erwarten, da immer mehr Regelungen im Hinblick auf ein netzdienlicheres Verhalten durch die Implementierung spannungseinprägender Verfahren realisiert werden. Dies hat auch Auswirkung auf das Verhalten im Fehlerfall, speziell im Hinblick auf die in den Netzanschlussbedingungen geforderte dynamische Netzstützung durch Blindstromeinspeisung sowie auf das Stabilitätsverhalten bei generatornahen Fehlern. Diese Aspekte stellen aktuelle Forschungsthemen im Bereich der doppelt speisenden Generatoren dar.

Das Beherrschen von Fehlern in Netzen mit stark abnehmendem Fehlerstrom wie im Falle von die DFIG bzw. verallgemeinert, mit schwachen Einspeisungen aus einem stark überlagerten Netz, stellt die Schutztechnik vor besondere Herausforderungen. Bedingt durch den Stromverfall ist eine Reserveschutzfunktion auf reiner Überstrombasis nicht immer sichergestellt. Abhilfe für diese "weak infeed"-Problematik bringt eine zusätzliche (tief eingestellte) Überstromauslösung mit Unterspannungsfreigabe bzw. eine erweiterte Schutzfunktionalität mittels unterspannungsgesteuerter AnregeSelbsthaltung der Überstromanregung.

Schließlich soll noch darauf hingewiesen werden, dass das Verhalten bei unsymmetrischen Fehlern aufgrund des dabei auftretenden Gegensystems für die Anlagen die größte Herausforderung darstellt. Auf diesen Aspekt konnte im Rahmen dieser Arbeit nicht näher eingegangen werden, es handelt sich um ein aktuelles und praktisch wichtiges Forschungsthema.

Für die Berechnung der Kurzschlussströme nach den einschlägigen Normen sind einfache Modelle der elektrischen Betriebsmittel, die mit möglichst wenigen verfügbaren Daten auskommen, erforderlich. Diese beruhen auf Vereinfachungen, die durch die Beschränkung auf spezielle Stromwerte zu bestimmten Zeiten bzw. Zeitbereichen, die sog. charakteristischen Kurzschlussgrößen, möglich sind. Es wurde der aktuelle Stand der Nachbildung der WKW mit DFIG nach den Normen beschrieben und bewertet, sowie ein verbessertes Verfahren auf der Grundlage der Generator- und Transformatorparameter aus den Ersatzschaltplänen vorgestellt. Zur Validierung dieses Ansatzes wurden zahlreiche FRT-Test ausgewertet.

Funding Note Open access funding provided by Graz University of Technology.

Hinweis des Verlags Der Verlag bleibt in Hinblick auf geografische Zuordnungen und Gebietsbezeichnungen in veröffentlichten Karten und Institutsadressen neutral. 
Open Access Dieser Artikel wird unter der Creative Commons Namensnennung 4.0 International Lizenz veröffentlicht, welche die Nutzung, Vervielfältigung, Bearbeitung, Verbreitung und Wiedergabe in jeglichem Medium und Format erlaubt, sofern Sie den/die ursprünglichen Autor(en) und die Quelle ordnungsgemäß nennen, einen Link zur Creative Commons Lizenz beifügen und angeben, ob Änderungen vorgenommen wurden. Die in diesem Artikel enthaltenen Bilder und sonstiges Drittmaterial unterliegen ebenfalls der genannten Creative Commons Lizenz, sofern sich aus der Abbildungslegende nichts anderes ergibt. Sofern das betreffende Material nicht unter der genannten Creative Commons Lizenz steht und die betreffende Handlung nicht nach gesetzlichen Vorschriften erlaubt ist, ist für die oben aufgeführten Weiterverwendungen des Materials die Einwilligung des jeweiligen Rechteinhabers einzuholen. Weitere Details zur Lizenz entnehmen Sie bitte der Lizenzinformation auf http://creativecommons.org/licenses/by/4.0/deed.de.

\section{Literatur}

1. IRENA (2020): Renewable capacity statistics 2020 International Renewable Energy Agency. Abu Dhabi: IRENA.

2. E-Control Statistikbroschüre (2019). https://www.e-control.at/publikationen/statistik bericht.

3. IEC 61400-21-1:2019 (2019):- Wind energy generation systems - Part 21-1: Measu rement and assessment of electrical characteristics. Wind turbines.
4. IEC 60909-0:2016 (2016): Short-circuit currents in three-phase AC systems - Part 0: Calculation of currents.

5. DIN EN 60909-0 (VDE 0102):2016 (2016): Kurzschlussströme in Drehstromnetzen Teil 0: Berechnung der Ströme.

6. Kovács, K. P., Rácz, I. (1959): Transiente Vorgänge in Wechselstrommaschinen, Band II. Budapest: Verlag der Ungarischen Akademie der Wissenschaften.

7. DIN EN 61400-27-1 (VDE 0127 27-1):2016-05 (2016): Windenergieanlagen Teil 27 1: Elektrische Simulationsmodelle Windenergieanlagen

8. FGW TR 3-Bestimmung der elektrischen Eigenschaften von Erzeugungseinheiten und -anlagen, Speicher sowie für deren Komponenten am Mittel-, Hoch- und Höchstspannungsnetz

9. VDE-AR-N 4110 Anwendungsregel: 2018-11 (2018): Technische Regeln für den Anschluss von Kundenanlagen an das Mittelspannungsnetz und deren Betrieb (TAR Mittelspannung)

10. VDE-AR-N 4120 Anwendungsregel: 2018-11 (2018): Technische Regeln für den Anschluss von Kundenanlagen an das Hochspannungsnetz und deren Betrieb (TAR Hochspannung)

11. VDE-AR-N 4130 Anwendungsregel: 2018-11 (2018): Technische Regeln für den Anschluss von Kundenanlagen an das Höchstspannungsnetz und deren Betrieb (TAR Höchstspannung)

12. E-CONTROL Technische und organisatorische Regeln für Betreiber und Benutzer von Netzen (TOR).

13. Fickert, L. (2017): Schutztechnik (Skriptum). Graz: Technische Universität.

14. ALSTOM Network (2002): Protection \& Automation Guide. ISBN 2-9518589-0-6.

\section{Autoren}

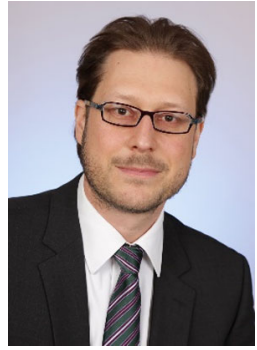

Robert Schürhuber

Prof. Schürhuber studierte an der Technischen Universität Wien Elektrotechnik und Technische Mathematik, wo er 2002 auch auf dem Gebiet der Theoretischen Elektrotechnik promovierte. Er war danach 15 Jahre in der Industrie in verschieden Bereichen der Energietechnik tätig, unter anderem befasste er sich der Steuerung und Regelung thermischer Anlagen, mit Erregereinrichtungen für Generatoren sowie dem elektrischen Umfeld von Großwasserkraftanlagen. Im Sommer 2017 wurde er an die Technische Universität Graz berufen und leitet dort seit Oktober 2017 das Institut für Elektrische Anlagen und Netze.

\section{Bernd R. Oswald}

Univ.-Prof. Dr.-Ing. habil. Bernd R. Oswald (79) studierte Elektrotechnik (Elektrische Maschinen und Antriebe) an der TU Dresden, wo er auch promoviert wurde und sich habilitierte. 1987-1992 Hochschuldozent an der TH Leipzig. 1992 Professorenamt neuen Rechts und Gründungsdekan des neuen Fachbereichs Elektrotechnik an der TH Leipzig. 1992 bis 2007 Professur Elektrische Energieversorgung an der Universität Hannover mit dem Forschungsschwerpunkt Netzausbau und -berechnung. 2003 bis 2015 Chairman von IEC TC 73 "Short-Circuit Currents" und seit 2004 Obmann von DKE K 121 und UK 121.1 „Kurzschlussströme”.

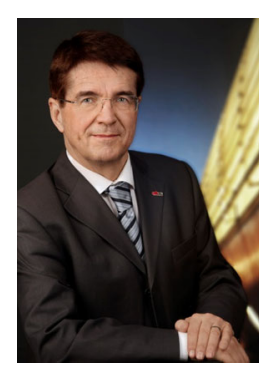

Lothar Fickert

Lothar H. Fickert promovierte 1974 an der Technischen Universität Wien und arbeitete danach 25 Jahre lang in der Industrie als Schutz- und Anlagentechniker in Netzplanung und Betrieb in der Energiewirtschaft (ELIN UNION, BROWN BOVERI, WIENSTROM). Seit 1998 wirkt er als Professor und Vorstand des Institutes für Elektrische Anlagen und Netze an der Technischen Universität Graz mit den Forschungsschwerpunkten elektrische Energiesysteme, Versorgungssicherheit und Schutztechnik. Er arbeitet in verschiedenen nationalen und internationalen Organisationen (IEC, CIGRE, CIRED, VDE, ÖVE usw.) mit und hat 9 Patente. Seit Oktober 2017 ist er Professor emeritus und widmet sich neben seiner Kernprofession, der Schutztechnik, auch aktuellen Sternpunktfragen sowie modernen Lehr- und Lernmedien („Flipped Classroom”).

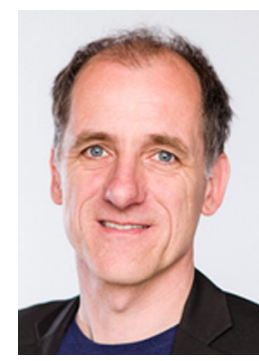

\section{Jens Fortmann}

Jens Fortmann erhielt sein Diplom in Elektrotechnik von der Technischen Universität Berlin im Jahr 1996 und promovierte an der Universität Duisburg-Essen im Jahr 2013. Von 1995 bis 2015 arbeitete er an der Modellierung, dem Kontrolldesign und der Netzintegration von Windenergieanlagen bei verschiedenen WEA-Herstellern. Seit 2015 ist er Professor an der HTW Berlin für Windenergie und Netzintegration. Seine Forschungsinteressen umfassen Regelung, Modellierung und Netzintegration von Erneuerbarer Energien. Er ist der Leiter der FGW-Arbeitsgruppe, die die deutsche Richtlinie TR4 zur Modellierung und Modellvalidierung der elektrischen Eigenschaften von WEA erstellt. 